Quim. Nova, Vol. 34, No. 8, 1472-1475, 2011

\title{
AVALIAÇÃO DO EFEITO DE GASES POLUENTES NA CORROSÃO METÁLICA: UM EXPERIMENTO PARA O ENSINO DA CORROSÃO
}

\author{
Bárbara Bidoia Bidetti, Priscila Aoki Balthazar, Heloisa Andréa Acciari e Eduardo Norberto Codaro* \\ Departamento de Física e Química, Faculdade de Engenharia de Guaratinguetá, Universidade Estadual de São Paulo, Av. Dr. \\ Ariberto Pereira da Cunha, 333, 12516-410 Guaratinguetá - SP, Brasil
}

Recebido em 27/10/10; aceito em 30/3/11; publicado na web em 10/6/11

\begin{abstract}
EVALUATION OF THE EFFECT OF GASEOUS POLLUTANTS IN METALLIC CORROSION: AN EXPERIMENT FOR TEACHING OF CORROSION. In this work one proposes a didactic experience to simulate atmospheric corrosion of copper and nickel, due to sulfur dioxide presence. This is an opportunity to understand some basic aspects of atmospheric corrosion, by using fundamental concepts in chemistry, reactions of extraction and characterization of pollutants, as well as their participation in corrosion process. This subject opens a space for discussion about necessity of pollutant gases emissions control for preservation of materials and the environment.
\end{abstract}

Keywords: chemistry education; atmospheric corrosion; sulfur dioxide.

\section{INTRODUÇÃO}

A corrosão atmosférica é o tipo de corrosão mais frequente em materiais metálicos expostos à intempérie. A atmosfera é uma mistura gasosa de composição aproximadamente constante, exceto no vapor de água e nos poluentes, que dependem da zona geográfica (tropical, subtropical, temperada, ártica ou antártica), da região (marinha, industrial, urbana, rural, árida) e estação do ano.

Os fatores naturais que influenciam a ação corrosiva da atmosfera são três: a temperatura, a chuva e o vento. As variações de temperatura entre o material metálico e a atmosfera (insolação) alteram o grau de molhamento e o tempo de secagem da superfície. A duração e os ciclos de chuva podem diminuir ou aumentar a velocidade de corrosão. Diminui, quando lava a superfície de impurezas sólidas (particulados) e aumenta, quando arrasta produtos que oferecem proteção. Com relação ao vento, além de seu poder erosivo, as variações na direção e na velocidade do vento alteram a composição relativa dos constituintes corrosivos da atmosfera. ${ }^{1}$

Os poluentes gasosos de origem antropogênica mais preocupantes, tanto em quantidade como em agressividade, são $\mathrm{SO}_{2}$, $\mathrm{SO}_{3}, \mathrm{NO}$ e $\mathrm{NO}_{2}$, sendo o primeiro o mais abundante em regiões urbanas e industriais. Estes gases são provenientes da queima de combustíveis derivados do petróleo, seja em fornos, fornalhas, incineradores ou motores de combustão interna e, uma vez em contato com a umidade do ar, formam soluções ácidas. ${ }^{2}$ Quando acontece precipitação, se o $\mathrm{pH}$ cai para valores menores que 5,6 a chuva é chamada de ácida. Embora nas regiões industrializadas o $\mathrm{pH}$ se encontre normalmente entre 4,0 e 5,6, na Europa e nos Estados Unidos já foram registrados valores menores que 3,0. ${ }^{3,4} \mathrm{O}$ desenvolvimento socioambiental e os fenômenos climáticos tornam a atmosfera um meio variável com condições difíceis de reproduzir em um laboratório. ${ }^{4,5}$

Os ensaios de corrosão atmosférica consistem em submeter diferentes materiais a vapores e gases dentro de câmaras fechadas durante dias, com o intuito de avaliar a resistência à corrosão num

*e-mail: codaro@feg.unesp.br ambiente que possa simular a agressividade da atmosfera na qual esses materiais serão expostos posteriormente em serviço. ${ }^{6}$ Neste contexto, o objetivo desta experiência é demonstrar de maneira simples e clara a deterioração da superfície de quatro materiais, de ampla aplicação tecnológica, como $\mathrm{Cu}, \mathrm{Zn}$, aço galvanizado e $\mathrm{Ni}$, na presença de $\mathrm{SO}_{2}$ e vapor de $\mathrm{H}_{2} \mathrm{O}$. Espera-se também conscientizar o aluno sobre as consequências indesejáveis do crescimento industrial e da importância da preservação do meio ambiente e dos materiais.

\section{FUNDAMENTAÇÃO TEÓRICA}

Dióxido de enxofre é um gás incolor de odor sufocante mais denso que o ar. No laboratório, pode ser preparado por aquecimento forte de enxofre, sulfetos ou dissulfetos, como representado pelas Equações 1-3:

$$
\begin{gathered}
\mathrm{S}(\mathrm{s})+\mathrm{O}_{2}(\mathrm{~g}) \rightarrow \mathrm{SO}_{2}(\mathrm{~g}) \\
4 \mathrm{FeS}(\mathrm{s})+7 \mathrm{O}_{2}(\mathrm{~g}) \rightarrow 2 \mathrm{Fe}_{2} \mathrm{O}_{3}(\mathrm{~s})+4 \mathrm{SO}_{2}(\mathrm{~g}) \\
4 \mathrm{FeS}_{2}(\mathrm{~s})+11 \mathrm{O}_{2}(\mathrm{~g}) \rightarrow 2 \mathrm{Fe}_{2} \mathrm{O}_{3}(\mathrm{~s})+8 \mathrm{SO}_{2}(\mathrm{~g})
\end{gathered}
$$

Este gás também pode ser obtido em condições menos enérgicas, pela adição de um ácido não oxidante ao sulfito ou hidrogenossulfito de sódio, segundo a Equação 4:

$$
\mathrm{NaHSO}_{3}(\mathrm{~s})+\mathrm{H}^{+}(\mathrm{aq}) \rightarrow \mathrm{Na}^{+}(\mathrm{aq})+\mathrm{SO}_{2}(\mathrm{~g})+\mathrm{H}_{2} \mathrm{O}(\mathrm{l})
$$

O gás pode ser identificado pela: reação com solução de dicromato de potássio, devido à formação de um aquocomplexo de cromo (III) de coloração azul esverdeada (Equação 5); pela reação com solução de iodato de potássio, devido à formação de iodo de coloração marrom (Equação 6) e pela reação com solução de cloreto de bário, devido à formação de um precipitado branco de sulfito de bário (Equação 7). Este precipitado é lentamente oxidado a sulfato sem mudanças significativas de cor (Equação 8). ${ }^{7}$ 


$$
\begin{aligned}
3 \mathrm{SO}_{2}(\mathrm{~g}) & +\mathrm{Cr}_{2} \mathrm{O}_{7}{ }^{2-}(\mathrm{aq})+11 \mathrm{H}_{2} \mathrm{O}(\mathrm{l})+2 \mathrm{H}^{+}(\mathrm{aq}) \rightarrow \\
& \rightarrow 2 \mathrm{Cr}\left(\mathrm{H}_{2} \mathrm{O}\right)_{6}{ }^{3+}(\mathrm{aq})+3 \mathrm{SO}_{4}{ }^{2-}(\mathrm{aq})
\end{aligned}
$$

$$
\begin{gathered}
5 \mathrm{SO}_{2}(\mathrm{~g})+2 \mathrm{IO}_{3}^{-}(\mathrm{aq})+4 \mathrm{H}_{2} \mathrm{O}(\mathrm{l}) \rightarrow \mathrm{I}_{2}(\mathrm{~s})+5 \mathrm{SO}_{4}{ }^{2-}(\mathrm{aq})+8 \mathrm{H}^{+}(\mathrm{aq})(6) \\
\mathrm{SO}_{2}(\mathrm{~g})+\mathrm{Ba}^{2+}(\mathrm{aq})+\mathrm{H}_{2} \mathrm{O}(\mathrm{l}) \rightarrow \mathrm{BaSO}_{3}(\mathrm{~s})+2 \mathrm{H}^{+}(\mathrm{aq}) \\
2 \mathrm{BaSO}_{3}(\mathrm{~s})+\mathrm{O}_{2}(\mathrm{~g}) \rightarrow 2 \mathrm{BaSO}_{4}(\mathrm{~s})
\end{gathered}
$$

Alguns gases podem ser coletados por deslocamento de água no dispositivo mostrado na Figura 1. Esses gases não devem reagir com a água e ser insolúveis nela, que não é o caso do $\mathrm{SO}_{2}$. No entanto, usando recipientes pequenos para diminuir o volume morto (espaço livre no balão e na mangueira) e condições controladas de temperatura para acelerar e completar a reação, é possível coletar de forma rápida um volume significativo de $\mathrm{SO}_{2}$. Desta maneira, posicionando corpos de prova metálicos na parte superior da proveta consegue-se atacar os mesmos numa fase gasosa constituída, principalmente, por $\mathrm{SO}_{2}$ e vapor de $\mathrm{H}_{2} \mathrm{O}$.

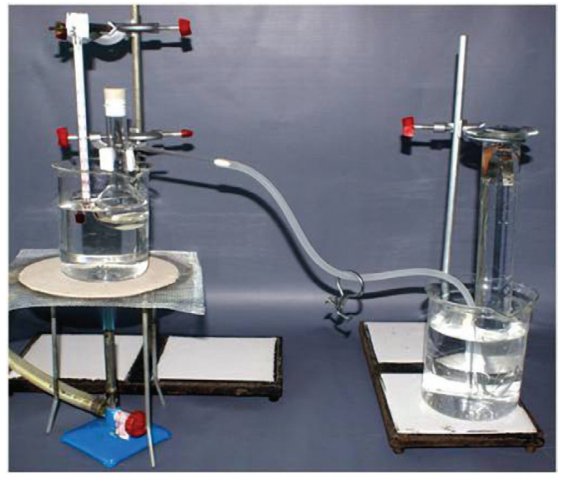

Figura 1. Dispositivo para coleta de gás $\mathrm{SO}_{2}$

A partir da dissolução de dióxido de enxofre em água, seja líquida ou na forma de vapor, pode-se obter diferentes espécies segundo o $\mathrm{pH}$ : $\mathrm{SO}_{3}{ }^{2-}(\mathrm{pH}>6,5), \mathrm{HSO}_{3}^{-}(\mathrm{pH} 1,5-6,5)$ e $\mathrm{SO}_{2} .2 \mathrm{H}_{2} \mathrm{O}(\mathrm{pH}$ $<1,5) .{ }^{2}$ Tanto ácido sulfuroso como seus sais são agentes redutores que formam ácido sulfúrico e sulfatos $\left[\mathrm{E}^{0}\left(\mathrm{SO}_{4}^{2-} / \mathrm{H}_{2} \mathrm{SO}_{3}\right)=+0,17 \mathrm{~V}\right]$, quando oxidados por oxigênio, peróxido de hidrogênio e outros agentes oxidantes semelhantes. Algumas das reações propostas na literatura $^{1}$ para a formação das soluções ácidas são mostradas nas Equações de 9 a 12:

$$
\begin{gathered}
\mathrm{SO}_{2}(\mathrm{~g})+\mathrm{H}_{2} \mathrm{O}(\mathrm{l}) \leftrightarrow \mathrm{H}^{+}(\mathrm{aq})+\mathrm{HSO}_{3}^{-}(\mathrm{aq}) \\
\mathrm{SO}_{2}(\mathrm{~g})+1 / 2 \mathrm{O}_{2}(\mathrm{~g})+\mathrm{H}_{2} \mathrm{O}(\mathrm{l}) \rightarrow \mathrm{H}^{+}(\mathrm{aq})+\mathrm{HSO}_{4}^{-}(\mathrm{aq}) \\
\mathrm{SO}_{2}(\mathrm{~g})+1 / 2 \mathrm{O}_{2}(\mathrm{~g}) \rightarrow \mathrm{SO}_{3}(\mathrm{~g}) \\
\mathrm{SO}_{3}(\mathrm{~g})+\mathrm{H}_{2} \mathrm{O}(\mathrm{l}) \leftrightarrow \mathrm{H}^{+}(\mathrm{aq})+\mathrm{HSO}_{4}^{-}(\mathrm{aq})
\end{gathered}
$$

Em atmosferas não poluídas, muitos metais de transição reagem com oxigênio formando principalmente óxidos ou hidróxidos, dependendo da umidade relativa do ar. Na presença de dióxido de enxofre esses produtos podem ser transformados em sulfatos bási$\cos$ (Equações 13 e 14). Em tempos prolongados de exposição ou concentrações elevadas deste gás, a reação pode levar diretamente à formação de sulfatos (Equações 15, 16 e 17). ${ }^{1,7-9}$

$$
\mathrm{Cu}_{2} \mathrm{O}(\mathrm{s})+\mathrm{SO}_{2}(\mathrm{~g})+\mathrm{O}_{2}(\mathrm{~g})+\mathrm{H}_{2} \mathrm{O}(\mathrm{l}) \rightarrow \mathrm{CuSO}_{4} \cdot \mathrm{Cu}(\mathrm{OH})_{2}(\mathrm{~s})
$$

$$
\begin{gathered}
\mathrm{Zn}(\mathrm{OH})_{2}(\mathrm{~s})+1 / 4 \mathrm{SO}_{2}(\mathrm{~g})+1 / 8 \mathrm{O}_{2}(\mathrm{~g}) \rightarrow \\
\rightarrow \mathrm{Zn}(\mathrm{OH})_{3 / 2}\left(\mathrm{SO}_{4}\right)_{1 / 4}(\mathrm{~s})+1 / 4 \mathrm{H}_{2} \mathrm{O}(\mathrm{l}) \\
\mathrm{Cu}(\mathrm{s})+\mathrm{SO}_{2}(\mathrm{~g})+\mathrm{O}_{2}(\mathrm{~g})+\mathrm{nH}_{2} \mathrm{O}(\mathrm{l}) \rightarrow \mathrm{CuSO}_{4} \cdot \mathrm{nH}_{2} \mathrm{O}(\mathrm{s}) \\
\mathrm{Zn}(\mathrm{s})+\mathrm{SO}_{2}(\mathrm{~g})+\mathrm{O}_{2}(\mathrm{~g})+\mathrm{nH}_{2} \mathrm{O}(\mathrm{l}) \rightarrow \mathrm{ZnSO}_{4} \cdot \mathrm{nH}_{2} \mathrm{O}(\mathrm{s}) \\
\mathrm{Ni}(\mathrm{s})+\mathrm{SO}_{2}(\mathrm{~g})+\mathrm{O}_{2}(\mathrm{~g})+\mathrm{nH}_{2} \mathrm{O}(\mathrm{l}) \rightarrow \mathrm{NiSO}_{4} \cdot \mathrm{nH}_{2} \mathrm{O}(\mathrm{s})
\end{gathered}
$$

Os materiais selecionados para esta experiência estão expostos às mais diversas condições atmosféricas, pela sua utilização em equipamentos, estruturas, instalações externas e ornatos. Cobre e suas ligas estão presentes em monumentos, estátuas e placas de homenagens. Também são fabricados tubos e telhas para a indústria civil e condutores para a indústria eletroeletrônica. Zinco e suas ligas são utilizados como revestimentos protetores de aços estruturais nas indústrias civil e automobilística. Aço galvanizado é usado na fabricação de telhas, calhas, rufos, coifas e dutos. Níquel e suas ligas são utilizados como revestimentos de aços e ligas de alumínio para a indústria aeronáutica, cujo principal interesse é a resistência à corrosão sob tensão e ao desgaste numa ampla faixa de temperaturas.

\section{MATERIAIS E REAGENTES}

Os materiais e os reagentes necessários para a realização deste experimento são: corpos de prova (c-d-p): sugerem-se duas chapas finas de cada material, de $70 \times 10 \mathrm{~mm}$, com um furo em cada extremo para amarração com fio de náilon; 2 suportes universais; 1 garra metálica; 1 balão de Engler de $100 \mathrm{~mL}$ com rolha; 1 mangueira de látex; 1 tubo de vidro em U; 1 pinça de Mohr; 1 tripé metálico; 1 tela de amianto; 1 bico de Bunsen; 1 anel metálico; 3 provetas, uma de 10 e duas de $250 \mathrm{~mL} ; 3$ béqueres, um de 600 e dois de $1000 \mathrm{~mL} ; 3$ tubos de ensaio; 1 estante para tubos de ensaio; 3 pipetas graduadas de 2,0 mL; 1 bastão de vidro; 1 funil de vidro; 4 vidros de relógio; 1 termômetro graduado de 0 a $100{ }^{\circ} \mathrm{C} ; 1$ seringa descartável de 20,0 $\mathrm{mL}$, com a ponta acoplada a um tubo fino de plástico de $20 \mathrm{~cm}$, para insuflar ar; 1 balança analítica; 1 pHmetro de bancada; 2 pissetas, uma com água destilada e outra com álcool; 1 lixa d'água $n^{\circ}$ 80; 1 pinça de madeira; 1 pinça metálica; 1 secador de cabelos; luvas; acetona; algodão; papel de filtro comum; $\mathrm{NaHSO}_{3}$ P.A.; solução de $\mathrm{HCl}$ 1,5 $\mathrm{mol} / \mathrm{L}$; solução de $\mathrm{K}_{2} \mathrm{Cr}_{2} \mathrm{O}_{7} 0,5 \mathrm{~mol} / \mathrm{L}$; solução de $\mathrm{KIO}_{3} 0,5 \mathrm{~mol} / \mathrm{L}$; solução de $\mathrm{BaCl}_{2} 0,5 \mathrm{~mol} / \mathrm{L}$ e água destilada.

\section{METODOLOGIA}

Para a realização da experiência é necessário dispor de uma ou mais capelas para exaustão de gases e também formar tantos grupos de alunos quantas forem as capelas disponíveis no laboratório. A primeira parte da experiência consistiu em evidenciar a formação do $\mathrm{SO}_{2}$, já que este gás é incolor. Para este propósito, foram utilizados dois tubos de ensaio contendo a ponta de uma espátula de $\mathrm{NaHSO}_{3}$. Foi preparada uma tira de papel de filtro umedecida com solução de $\mathrm{K}_{2} \mathrm{Cr}_{2} \mathrm{O}_{7} 0,5 \mathrm{~mol} / \mathrm{L}$, foram adicionados $2,0 \mathrm{~mL}$ de solução de $\mathrm{HCl} 1,5$ $\mathrm{mol} / \mathrm{L}$ em um dos tubos com a tira posicionada na boca do mesmo. A solução foi aquecida suavemente e observada a mudança na coloração do papel para azul esverdeado, de acordo com a Equação 5. No outro tubo o mesmo procedimento foi realizado, utilizando uma tira de papel de filtro umedecida com solução de $\mathrm{KIO}_{3} 0,5 \mathrm{~mol} / \mathrm{L} \mathrm{e}$ foi observada a mudança de coloração do papel para marrom, de acordo com a Equação 6.

Para a segunda parte da experiência - produção e coleta de $\mathrm{SO}_{2}$ - cada grupo deverá trabalhar com quatro c-d-p, dois de um material e dois de outro. A preparação da superfície dos c-d-p, exceto aço 
galvanizado, foi feita com o auxílio de uma lixa com o intuito de obter as duas faces brilhantes e fisicamente semelhantes. Após esse procedimento, o c-d-p foi lavado com água destilada e depois com álcool e seco com o secador de cabelos. Os extremos dos c-d-p foram amarrados com fios de náilon, a fim de tornar possível puxá-los do fundo da proveta no final do experimento. O primeiro c-d-p foi introduzido na proveta flexionando-o na forma de um U e empurrado com um bastão de vidro para dentro da proveta, sem encostá-lo no fundo. A seguir, o segundo c-d-p foi flexionado e posicionado na boca da proveta, de forma cruzada ao primeiro c-d-p, e empurrado até ficar a aproximadamente $1 \mathrm{~cm}$ deste último. Com este arranjo, não existe risco de gotejamento por condensação nem contato entre os produtos de corrosão dos c-d-p. Este procedimento foi repetido em outra proveta que serviu de branco. As provetas foram preenchidas com água destilada, invertidas e introduzidas em dois béqueres contendo aproximadamente $700 \mathrm{~mL}$ de água destilada cada um, conforme mostrado na Figura 1.

Em seguida, pesou-se 1,0 g de $\mathrm{NaHSO}_{3}$ e colocou-se dentro do balão. Assegurou-se que o nível da água no béquer do banho cobrisse metade do balão. Utilizando uma proveta, mediu-se 7,0 mL de solução de $\mathrm{HCl}$ 1,5 mol/L. Colocou-se a solução de ácido dentro do balão com o auxilio de um funil e fechou-se rapidamente com a rolha. Como à temperatura ambiente a reação é lenta, o risco de perda de gás por vazamento é baixo. $\mathrm{O}$ banho-maria foi aquecido a uma taxa de 1 a $2{ }^{\circ} \mathrm{C} / \mathrm{min}$ até uma temperatura entre 60 e $65^{\circ} \mathrm{C}$. Essa temperatura foi mantida até o consumo total do $\mathrm{NaHSO}_{3}$. Nestas condições, o $\mathrm{SO}_{2}$ deslocou entre 70 e $80 \mathrm{~mL}$ de água na proveta invertida. Em seguida, retirou-se o tubo em $\mathrm{U}$ do interior da proveta. Na outra proveta, que serviu de branco, insuflou-se com uma seringa um volume de ar igual àquele deslocado pelo $\mathrm{SO}_{2}$. Este branco representa a exposição dos materiais numa atmosfera não poluída. Logo após, mediu-se o $\mathrm{pH}$ da água nos béqueres com as provetas e os dois conjuntos foram guardados por um período de 3 a 7 dias. Após esse período mediram-se os pHs novamente. Com uma pipeta foram retirados 2,0 $\mathrm{mL}$ de água de cada um dos béqueres, transferidos a dois tubos de ensaio e gotejada solução de $\mathrm{BaCl}_{2}$ 0,5 mol/L para detectar a presença de sulfito ou sulfato, segundo as Equações 7 e 8 . Dentro da capela, os c-d-p foram retirados das provetas puxando-se os fios de náilon, colocados em vidros de relógio, secados ao ar e comparados visualmente. Para finalizar, as soluções utilizadas na primeira e segunda parte da experiência foram transferidas para frascos de resíduos.

\section{DISCUSSÃO}

Nesta experiência pode-se constatar o efeito do $\mathrm{SO}_{2}$ na corrosão de $\mathrm{Cu}, \mathrm{Zn}$, aço galvanizado e Ni. Os c-d-p expostos a uma mistura de ar e vapor de $\mathrm{H}_{2} \mathrm{O}$ permaneceram praticamente intactos, Figura 2a,c, enquanto que aqueles expostos a uma mistura de $\mathrm{SO}_{2}$ e vapor de $\mathrm{H}_{2} \mathrm{O}$ foram corroídos, Figura $2 b$,d. As superfícies do $\mathrm{Cu}, \mathrm{Zn}$, aço galvanizado e Ni exibiram aspecto opaco com produtos de corrosão heterogeneamente distribuídos. Os produtos obtidos sobre o $\mathrm{Cu}$ apresentaram colorações marrons e azuis celestes, sobre Zn e aço galvanizado foram brancas e sobre o $\mathrm{Ni}$ foram verdes e pretas. Com base na literatura ${ }^{1,7-9}$ e nos resultados obtidos, esses produtos devem estar constituídos principalmente por óxidos, sulfatos básicos e sulfatos. Por exemplo, $\mathrm{Cu}_{2} \mathrm{O}$ é vermelho, $\mathrm{CuO}$ é preto, $\mathrm{CuSO}_{4} \cdot \mathrm{nCu}(\mathrm{OH})_{2}(\mathrm{n}=$ 1 a 3 ) são azuis esverdeados, $\mathrm{CuSO}_{4} \cdot \mathrm{nH}_{2} \mathrm{O}(\mathrm{n}=1$ a 5) são azuis, $\mathrm{ZnO}$, $\mathrm{Zn}(\mathrm{OH})_{3 / 2}\left(\mathrm{SO}_{4}\right)_{1 / 4}(\mathrm{~s})$ e $\mathrm{ZnSO}_{4} \cdot \mathrm{nH}_{2} \mathrm{O}(\mathrm{n}=1$ a 7$)$ são brancos, $\mathrm{Ni}_{2} \mathrm{O}_{3}$ é preto, $\mathrm{NiO}$ e $\mathrm{NiSO}_{4} \cdot \mathrm{nH}_{2} \mathrm{O}(\mathrm{n}=1$ a 7$)$ são verdes. Neste contexto, fica evidente a presença de uma mistura de produtos de corrosão sobre a superfície desses metais. Dentre estes produtos, é atribuída aos óxidos a formação de uma película fina e aderente resistente à corrosão, no entanto, os sulfatos básicos, por serem insolúveis, também podem conferir certa proteção atuando como barreira entre o material e o meio. ${ }^{10,11}$
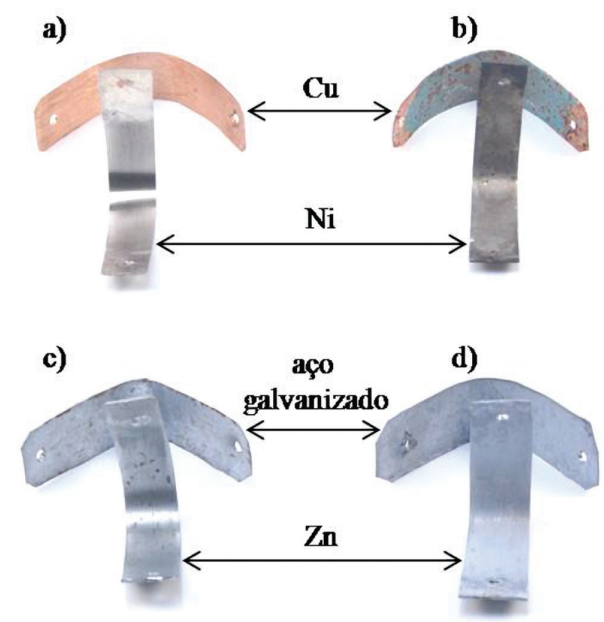

Figura 2. C-d-p após 7 dias de exposição em: a) e c) ar + vapor de $\mathrm{H}_{2} \mathrm{O}, \mathrm{b}$ e d) $\mathrm{SO}_{2}+$ vapor de $\mathrm{H}_{2} \mathrm{O}$

Considerando os potenciais padrão de redução, ${ }^{8} \mathrm{E}^{0}\left(\mathrm{Cu}^{2+} / \mathrm{Cu}\right)$ $=+0,34 \mathrm{~V}, \mathrm{E}^{0}\left(\mathrm{Zn}^{2+} / \mathrm{Zn}\right)=-0,76 \mathrm{~V}$ e $\mathrm{E}^{0}\left(\mathrm{Ni}^{2+} / \mathrm{Ni}\right)=-0,25 \mathrm{~V}$, somente $\mathrm{Zn}$ e Ni deveriam ser atacados em meio ácido. Apesar do $\mathrm{Fe}$ do aço ter um valor negativo de potencial, $\mathrm{E}^{0}\left(\mathrm{Fe}^{2+} / \mathrm{Fe}\right)=-0,44 \mathrm{~V}$, o aço está protegido pelo $\mathrm{Zn}$ por dois mecanismos: proteção por barreira, exercida pela camada de $\mathrm{Zn}$ que se interpõe entre o aço e o meio e, proteção catódica, em que o $\mathrm{Zn}$ é oxidado preferencialmente ao aço quando ocorre uma eventual falha nessa camada. No caso do $\mathrm{Cu}$, a sua oxidação é termodinamicamente desfavorável, no entanto, na presença de $\mathrm{O}_{2}\left[\mathrm{E}^{0}\left(\mathrm{O}_{2} / \mathrm{H}_{2} \mathrm{O}\right)=+1,23 \mathrm{~V}\right]$ o ataque do $\mathrm{Cu}$ se torna possível (Equação 18).

$$
\begin{gathered}
2 \mathrm{Cu}(\mathrm{s})+\mathrm{O}_{2}(\mathrm{~g})+4 \mathrm{H}^{+}(\mathrm{aq}) \rightarrow \\
\rightarrow 2 \mathrm{Cu}^{2+}(\mathrm{aq})+2 \mathrm{H}_{2} \mathrm{O}(\mathrm{l}) \Delta \mathrm{E}^{0}=+0,89 \mathrm{~V}
\end{gathered}
$$

As medidas de $\mathrm{pH}$ realizadas após o experimento indicaram uma diminuição apreciável (cerca de 4 unidades) somente na água onde o $\mathrm{SO}_{2}$ foi coletado. $\mathrm{O}$ valor do $\mathrm{pH}$ não mudou significativamente durante 7 dias, estabilizando-se em torno de 2,70. Este fato revela o caráter ácido do $\mathrm{SO}_{2}$ (Equação 9) e sua elevada solubilidade à temperatura ambiente. O teste com $\mathrm{BaCl}_{2}$ nesta água indicou a presença de $\mathrm{SO}_{3}{ }^{2-}$ ou $\mathrm{SO}_{4}^{2-}$ mediante a formação de um precipitado branco (Equações 7 e 8). Cabe destacar que a solubilidade dos sais $\mathrm{BaSO}_{3} \mathrm{e} \mathrm{BaSO}_{4}$ aumenta quando o $\mathrm{pH}$ diminui, devido ao deslocamento dos equilíbrios nas Equações 9 e 12 no sentido dos reagentes, resultando na diminuição da concentração dos ânions $\mathrm{HSO}_{3}^{-} \mathrm{e} \mathrm{HSO}_{4}^{-} \mathrm{e}$, consequentemente, do $\mathrm{SO}_{3}{ }^{2-}$ e $\mathrm{SO}_{4}{ }^{2-}$. Se o meio for moderadamente ácido a reação mencionada se manifesta com o aparecimento de um precipitado, mas se o meio for fortemente ácido $(\mathrm{pH}<1,5)$ isto pode não acontecer porque os produtos iônicos, $\left[\mathrm{Ba}^{2+}\right]\left[\mathrm{SO}_{3}{ }^{2-}\right]$ e $\left[\mathrm{Ba}^{2+}\right]\left[\mathrm{SO}_{4}{ }^{2-}\right]$, não ultrapassam os valores dos respectivos Kps. ${ }^{7}$

\section{CONSIDERAÇÕES FINAIS}

Com esta experiência é possível detectar a presença do $\mathrm{SO}_{2}$, constatar seu efeito corrosivo e demonstrar seu caráter ácido. O método de obtenção e de caracterização é simples e executável com o material comum de laboratório. Revela de forma clara a deterioração de materiais metálicos de ampla aplicação tecnológica. Esta experi- 
ência também abre um espaço para a discussão sobre a necessidade do controle das emissões de gases poluentes para a preservação dos materiais.

\section{AGRADECIMENTOS}

Ao CNPq, FUNDUNESP e PROEX/UNESP.

\section{REFERÊNCIAS}

1. Gentil, V.; Corrosão, $5^{\text {a }}$ ed., LTC: Rio de Janeiro, 2007.

2. Martins, C. R.; de Andrade, J. B.; Quim. Nova 2002, 25, 259.

3. Baird, C.; Química Ambiental, 2a ed., Bookman: Porto Alegre, 2005.

4. Carvalho Jr., V. N.; Revista Tecnologia 2004, 2, 61.
5. Magliavacca, D. M.; Teixeira, E. C.; Machado, A. C. M.; Pires, M. R.; Quim. Nova 2005, 28, 371.

6. Gemelli, E.; Corrosão de Materiais Metálicos e sua Caracterização, $1^{\mathrm{a}}$ ed., LTC: Rio de Janeiro, 2001.

7. Vogel, A. I.; Química Analítica Qualitativa, $5^{\text {a }}$ ed., Mestre Jou: São Paulo, 1981

8. Talbot, D. E. J.; Talbot, J. D. R.; Corrosion Science and Technology, $1^{\text {st }}$ ed, CRC Press LLC: New York, 1998.

9. Panossian, Z.; Corrosão e Proteção Contra Corrosão em Equipamentos e Estruturas Metálicas, $1^{\text {a }}$ ed., IPT: São Paulo, 1993.

10. Scully, J. C.; The Fundamentals of Corrosion, $2^{\text {nd }}$ ed., Pergamon Press: Oxford, 1975.

11. Craig, B. D.; Fundamental Aspects of Corrosion Films in Corrosion Science, Plenum Press: New York, 1991. 